\title{
Effectiveness and medication acceptance of olanzapine disintegrating tablets compared to standard olanzapine tablets in acutely treated psychiatric patients
}

\author{
Jörg Czekalla' \\ Thomas Wagner ${ }^{2}$ \\ Alexander Schacht ${ }^{2}$ \\ Michael Kluge ${ }^{3}$ \\ Bruce Kinon $^{4}$ \\ 'Department of Psychiatry and \\ Psychotherapy, RWTH Aachen \\ University, Germany; ${ }^{2}$ Medical \\ Department, Lilly Deutschland GmbH, \\ Bad Homburg, Germany; ${ }^{3}$ Max Planck \\ Institute for Clinical Psychiatry, \\ Munich, Germany; ${ }^{4}$ Lilly Research \\ Laboratories, Indianapolis, USA
}

\begin{abstract}
Background: This study compared effectiveness and acceptance of orally disintegrating olanzapine tablets (ODT) with standard-coated tablets (SOT) in acutely ill psychiatric patients admitted to psychiatric hospitals for emergency treatment.

Methods: Large, prospective, observational study at hospital emergency units across Germany in patients with a diagnosis or tentative diagnosis of acute schizophrenia treated with ODT or SOT. Clinical (CGI-S and CGI-I) outcomes, attitudes towards medication (Nursing Assessment of Medication Acceptance, NAMA) scale, suicidal ideation, and adverse events were assessed at start of treatment and after 2 weeks.

Results: Both olanzapine formulations, ODT $(\mathrm{N}=247)$ and SOT $(\mathrm{N}=207)$, showed similar effectiveness after 2 weeks. CGI-I improved in $92.1 \%$ of patients (ODT: $91.8 \%$, SOT: 92.3\%). In patients receiving both formulations suicidal ideations were reduced (ODT from $53.9 \%$ to $20.6 \%$, SOT from $51.2 \%$ to $22.7 \%$ ). ODT was preferably given to severely ill (SOT: $49.8 \%$, ODT: $64.4 \%$ ) and aggressive patients. Adverse events were reported for $6.5 \%$ of ODT- and $2.9 \%$ of SOT-patients. This difference was possibly caused by the characteristics of patients receiving ODT.
\end{abstract}

Conclusions: This non-randomized, observational study shows comparable outcomes and tolerability in patients treated with both olanzapine formulations. In an acute treatment setting, orally disintegrating tablets were preferably used for more severely ill and aggressive patients with low medication acceptance.

Keywords: olanzapine, acute treatment, schizophrenia, observational study

\section{Background}

Schizophrenia and related disorders are severe, incapacitating psychiatric diseases that, in most cases, lead to life-time significant burdens on patients as well as family members and the society in general (Torrey 1995). Medication non-compliance is a major concern in the treatment of acutely ill psychiatric patients. Studies indicate that treatment failure is often linked to non-compliance (Soskis 1978; Cooper et al 2007). Different application forms other than conventional tablets may help to improve a patient's compliance. New formulations of established antipsychotic medication have recently emerged that should extend the utility and flexibility of administration and treatment (Caine 2006). The orally disintegrating tablet (ODT) formulation of olanzapine (Zydis ${ }^{\circledR}$ in the US and Velotab ${ }^{\circledR}$ in Europe, available in Germany since 2000) may provide an alternative means of treating non-compliant patients as well as those patients, who are either unable or reluctant to swallow a tablet, as ODT is designed to dissolve upon contact with saliva. Corresponding formulations are available for other 
antipsychotics, eg, risperidone or aripiprazole. In a small study of schizophrenic patients, the mean disintegration time of ODT was 15.8 seconds, and subjective comments of the patients indicated high medication acceptance due to convenience of intake and subjective impression of effectiveness (Chue et al 2002; Bergstrom et al 2004). Removal of ODT from the tongue is also more difficult than for the standard (coated) olanzapine tablet (SOT) since it forms an amorphous residue that can only be removed by scraping the tongue. The use of orally disintegrating tablets in other indications (eg, migraine, depression), has been successfully tested in other studies (Dowson et al 2002; Behnke et al 2003). Improved compliance has, besides improved cognitive functioning and reduced negative symptoms, also been identified as a possible factor leading to a decrease in suicidality (Meltzer et al 1998). In comparison to haloperidol, olanzapine decreased the suicide rate after one year of treatment in a clinical trial (Glazer 1998). Little information is available on the effect of olanzapine on suicidal ideation from large naturalistic studies although safety and effectiveness of olanzapine in acutely ill and first-episode schizophrenic patients has been investigated in observational studies previously (Alvarez et al 2003; Bobes et al 2003). In the present study, we aimed to evaluate the use of ODT and SOT in a real-life setting with regard to patterns of use, patient types treated, treatment outcomes, and medication compliance. The effectiveness was assessed and documented by physicians using the Global Clinical Impression - Severity (CGI-S) and CGI - Improvement (CGI-I) scale as well as the MADRS suicidality item (Item \#10), and nursing global impression (NGI), and medication acceptance was assessed by nursing staff. The study duration was 2 weeks to document primarily the short-term effects of the pharmacological intervention by the initially treating physician and nursing staff team in acute psychiatric emergency wards. Since clinical trials are conducted using experimental conditions and results can often not be generalized to normal clinical practice, the present study was undertaken and conducted in a naturalistic setting.

\section{Methods}

\section{Patient population and study design}

This was a prospective, multicenter naturalistic study of standard (coated) olanzapine tablets (SOT) and olanzapine disintegrating tablets (ODT) in adult male or female patients arriving at the 24-hour emergency-service of psychiatric care hospitals with a diagnosis or tentative diagnosis of acute schizophrenia. The objective of this study was to observe the use of SOT and ODT with regard to effectiveness (defined as efficacy in the context of a natural setting), safety, and course of acute psychotic disease under naturalistic conditions.

The study involved 94 general and psychiatric hospitals located throughout Germany, and was conducted from October 2001 until July 2002. A median of 5 patients were documented per hospital (mean 5, range 1-15). Patients with hospital admission for other reasons than acute therapy (eg, transferred from another hospital subsequent to acute therapy or for elective adjustment of medication) were not eligible for participation. All data were collected anonymously and in full compliance with German Privacy Law regulations. All types of treatment observed were routinely available to German psychiatric emergency wards. The assessed medications were not provided by the manufacturer, but prescribed in the context of clinical routine care. As no specific treatments were classified for the observation, and the choice of therapy as well as switching between application forms was completely at the physician's discretion, this study reflects standard care in Germany. According to German legislation, no informed consent was needed for this observational study. For correct and complete documentation of data and for adverse event reporting, participating physicians received adequate reimbursement in accordance with the GOÄ (German physician fee schedule) in the amount of 150 per completely documented patient.

\section{Assessments}

Criteria for the evaluation of effectiveness were Clinical Global Impression - severity (CGI-S) (Guy 1976) at start of treatment and at final documentation (14 days), Clinical Global Impression - Improvement (CGI-I) at final documentation (14 days). Suicidality was assessed by the Montgomery Asberg Depression Rating Scale (MADRS) item 10 (suicidal ideation) (Montgomery and Asberg 1979) at baseline and at final documentation (14 days). CGI and MADRS item 10 were completed by the physician. Patients' medication acceptance and attitude towards olanzapine medication was assessed by the Nursing Assessment of Medication Acceptance (NAMA) questionnaire (Kinon et al 2003) to be completed by the nursing personnel at baseline and at final observation. Tolerability was assessed as the incidence and type of adverse events reported. Patient characteristics and medical history (type of disease) were also documented.

\section{Statistical analysis}

The statistical analysis was carried out using $\mathrm{SAS}^{\circledR}$, Version 8.2 (SAS Institute Inc). All efficacy parameters were evaluated based on the full analysis set (intent to treat population), 
ie, patients with at least one dose of olanzapine and at least one post baseline value available. Data are presented as means with their standard deviations (mean \pm SD) unless otherwise indicated. Furthermore, 95\% confidence intervals (CI) were calculated without any correction for multiplicity. Sample sizes may vary because of missing values for certain parameters.

It was planned to observe 480 patients treated with olanzapine. This allows detection of an adverse event with an incidence of $0.6 \%$ at least once during the observation with a probability of 0.95 . In order to compare the outcomes of patients treated with the two formulations after 2 weeks, the probability of having a better score (CGI-S, CGI-I, MADRS item 10) ODT vs SOT was calculated as relative effect using non-parametric methods (Brunner et al 2002). These methods are generalizations of the Wilcoxon-Mann and Whitney test (U-test) and the relative effect is the measure used by the U-Test. If one assumes Gaussian distribution of data, the relative effect is a function of the effect size, ie, a relative effect of $50 \%$ corresponds to an effect size of 0 standard deviations. A relative effect of more than $50 \%$ would indicate better scores for ODT compared to SOT at 2 weeks, whereas a relative effect below $50 \%$ would mean that the probability of observing a better score for ODT is lower. If Cohen's suggestions regarding small $(\mathrm{d}=0.2)$, medium $(\mathrm{d}=0.5)$, or large $(d=0.8)$ effect sizes in the case of normal distribution are applied to relative effects, a relative effect would be called small, medium, or large if they are larger than $0.556,0.635$, or 0.714, respectively (Brunner and Munzel 2002). Negative effect sizes of $\mathrm{d}=-0.2, \mathrm{~d}=-0.5$, and $\mathrm{d}=-0.8$ correspond to relative effects of $0.444,0.362$, and 0.286 , respectively.
The advantages of the relative effect over the effect size are the independence from the assumption of normal distribution, the correct description of ordered categorical data, and the interpretation as a simple probability. 95\% CIs for the relative effects were calculated (Brunner et al 2002). The relative effects for CGI-S and MADRS item 10 were calculated within strata defined by the respective baseline value to reduce the bias introduced by the non-randomized design. However, due to the small sample sizes in some strata it was not possible to adjust for further confounding variables (eg, study site).

\section{Results \\ Patient characteristics}

Overall, 456 patients were documented prospectively in this observational study. 32 patients $(7.0 \%)$ had discontinued the therapy prematurely (physician information), primarily because of a change of therapy (18/32), the occurrence of an adverse event (3/32), and for other reasons such as dismissal or referral to another hospital (11/32). The majority of patients was female (250 out of 456, 54.8\%). Patient demographics are summarized in Table 1.

Patients' age ranged from 18 to 93 years with a median age of 38 years, and with females on average being older than males. More than $80 \%$ of the patients documented $(366,80.3 \%)$ were treated because of schizophrenia or schizotypal and delusional disorders. Further underlying diseases reported were mood (affective) disorders (79, 17.3\%), organic disorders including symptomatic mental disorders $(45,9.9 \%)$, disorders of adult personality and behaviour

Table I Baseline characteristics of patients treated with olanzapine disintegrating tablets or standard olanzapine tablets

\begin{tabular}{|c|c|c|c|}
\hline \multicolumn{2}{|l|}{ Characteristic } & \multirow{2}{*}{$\begin{array}{l}\begin{array}{l}\text { SOT } \\
(\mathbf{N}=207)\end{array} \\
4 \mathrm{I} . \mathrm{I}( \pm 15.6)\end{array}$} & \multirow{2}{*}{$\begin{array}{l}\begin{array}{l}\text { ODT } \\
(\mathbf{N}=\mathbf{2 4 7})\end{array} \\
41.2( \pm 15.8)\end{array}$} \\
\hline Age (years) & Mean $( \pm S D)$ & & \\
\hline & Median & 38.0 & 38.0 \\
\hline & Range & $18.0-87.0$ & 18.0-93.0 \\
\hline \multirow[t]{2}{*}{ Sex, n (\%) } & Male & I0I (49.0\%) & $105(42.4 \%)$ \\
\hline & Female & $106(51.0 \%)$ & $142(56.8 \%)$ \\
\hline Duration of & Mean $( \pm S D)$ & $6.1( \pm 7.1)$ & $8.5( \pm 9.4)$ \\
\hline disease (years) & Median & 3.0 & 5.0 \\
\hline \multirow[t]{6}{*}{ n (\%) } & $\begin{array}{l}\text { Schizophrenia, schizotypal, } \\
\text { delusional }\end{array}$ & 159 (76.8\%) & $205(83.0 \%)$ \\
\hline & Mood (affective) disorder & $5 \mathrm{I}(24.6 \%)$ & $28(11.3 \%)$ \\
\hline & Organic & $20(9.7 \%)$ & $24(9.7 \%)$ \\
\hline & Disorders of adult personality & $18(8.7 \%)$ & $20(8.1 \%)$ \\
\hline & Mental and behavioral disorders & $10(4.8 \%)$ & II (4.5\%) \\
\hline & due to psychoactive substance abuse & & \\
\hline
\end{tabular}

Abbreviations: ODT, orally disintegrating olanzapine tablet; SOT, standard-coated olanzapine tablet; SD, standard deviation. 
(38, 8.3\%), and mental and behavioural disorders due to psychoactive substance abuse $(21,4.6 \%)$.

Physicians were free to decide which oral olanzapine formulation to use. For 207 (45.4\%) patients, SOT was documented as initial treatment, and 247 (54.2\%) patients started with ODT. For 2 patients, the olanzapine formulation used was not documented. No major differences were seen in the patients' demographic characteristics when comparing the two cohorts (see Table 1). However, the results indicate that patients' disease characteristics played a major role in determining the type of tablet given. Patients receiving ODT presented on average with a slightly longer mean duration of the disease. ODT was preferred for patients with agitated behaviour (externally-directed aggression, institutionalization, and psychomotor excitement), whereas the SOT was preferred for patients with psychomotor inhibition (see Figure 1).

\section{Dosage and concomitant medications}

The lowest daily dose documented was $2.5 \mathrm{mg}$, whereas the maximum daily dose was $40 \mathrm{mg}$ for SOT and $60 \mathrm{mg}$ for ODT. The average daily dose increased from $13.0 \pm 6.9 \mathrm{mg}$ at baseline to $16.9 \pm 7.8 \mathrm{mg}$ for SOT after the 2-week observational period. The mean dosage in patients receiving ODT started at $14.1 \pm 6.9 \mathrm{mg}$ at baseline and increased to $18.2 \pm 7.7 \mathrm{mg}$ after 2 weeks. The percentage of patients receiving high doses of olanzapine (ie, $\geq 20 \mathrm{mg}$ /day) increased from approximately one third at baseline to around $50 \%$ at the last daily dose documented in the study. At baseline, $24.2 \%$ SOT patients received a highdose, compared to $41.1 \%$ after 2 weeks of treatment. The proportion of ODT patients receiving a high-dose increased from $34.4 \%$ at baseline to $59.1 \%$ after 2 weeks.

Patients in both groups (SOT and ODT) had received antipsychotic medication before the study (43.5\% and 39.3\%, respectively). More than $75 \%$ of all acutely treated patients received a psychotropic co-medication during the observational study. The most commonly taken co-medications were benzodiazepines/tranquilizers ( $58.6 \%$ of patients), other antipsychotics (typicals $25.2 \%$, risperidone $0.7 \%$, quetiapine $0.7 \%$, amisulpride $0.2 \%$ ) and antidepressants $(19.1 \%$ of patients). Anticonvulsants were used in $8.1 \%$ of patients.

\section{Clinical Global Impression (CGI)}

The change in disease severity (CGI-S) is shown in Table 2 for patients treated with SOT vs ODT.

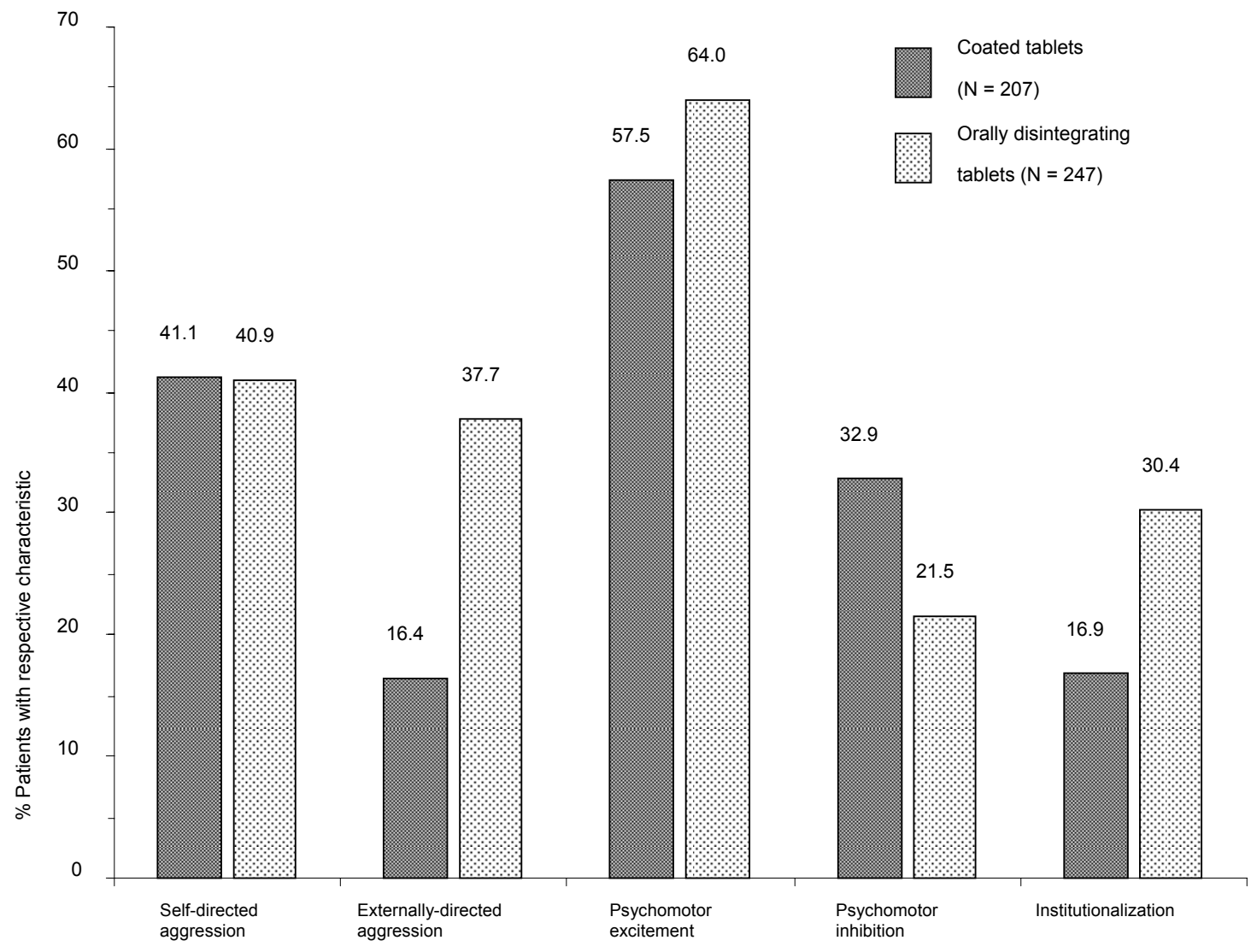

Figure I Patient baseline disease characteristics by application form (coated tablets vs orally disintegrating tablets). 
In patients receiving both formulations disease severity decreased, measured as CGI-S rated by the physician, independent of dosage group after 2 weeks of treatment. The proportion of patients treated with SOT who were at least severely ill was reduced from $49.8 \%$ at baseline to $5.3 \%$ after 2 weeks of treatment. The respective results for patients treated with ODT were $64.4 \%$ at baseline and $15.0 \%$ after 2 weeks (Table 2). In parallel, global impression of disease severity evaluated by the nursing staff (NGI-S) led to comparable results.

An improvement in disease severity from baseline (CGI-I categories improved, much improved, or very much improved) was documented for $92.1 \%$ of patients by the physicians $(91.8 \%$ for patients treated with SOT, $92.3 \%$ for patients treated with ODT). Assessment of improvement conducted by nursing staff (NGI-I) produced comparable results.

The proportion of patients with an improved disease severity (CGI-I) was comparable between low-dose $(<20$ $\mathrm{mg} /$ day, $91.5 \%$ ) and high-dose ( $\geq 20 \mathrm{mg} /$ day, $92.7 \%$ ) olanzapine treatment subgroups.

Relative effects analyses (ODT vs SOT) were performed on CGI-I and CGI-S data. The CGI-S analysis was stratified by CGI-S baseline severity. Relative effects of ODT compared to SOT for these parameters are summarized with $95 \%$ confidence intervals in Table 3.

A high $(>0.5)$ relative effect would indicate a better outcome for ODT treated patients compared to SOT treated patients. Generally, relative effects were close to 0.5 with $95 \% \mathrm{CI}$ encompassing 0.5 , indicating no differences in documented effectiveness in clinical practices. The only relative effect not encompassing 0.5 was seen for CGI-S in the 30 patients with extremely severe baseline disease (CGI-S $=7$ ) for which a lower relative effect for ODT was found. A larger proportion of these extremely acute patients receiving ODT were institutionalized and showed externally directed aggression $(50 \%, 11 / 22)$, whereas these characteristics were present in only $25 \%(2 / 8)$ of the SOT patients, respectively. These extremely ill and institutionalized/ externally aggressive patients had a lower probability for short-term treatment response.

\section{Suicidal thoughts}

Overall, there were $240(52.6 \%)$ patients with some degree of suicidal ideation at baseline as measured by MADRS item 10 , including 30 patients $(6.6 \%)$ with distinct suicidal plans. Data were missing for $12(2.6 \%)$ patients. Suicidality ratings decreased from baseline to final observation ( 2 weeks) in 189 out of 456 patients $(41.5 \%)$ and increased in only 2 patients $(0.4 \%)$. As Figure 2 shows, the proportion of patients with any suicidal thoughts decreased from $51.2 \%$ and $53.9 \%$, respectively at baseline to $22.7 \%$ and $20.6 \%$ after 2 weeks of treatment for SOT and ODT, respectively. No patients with distinct suicidal plans were observed after 2 weeks of treatment.

The non-parametric analysis using relative effects did not reveal consistent differences in suicidality rating improvements between patients treated with ODT and SOT.

Analysis of changes in suicidality by treatment dose revealed no apparent differences between patients receiving either high-dose or the low-dose olanzapine treatment. The proportion of patients with decreased suicidality was $44.2 \%$ after 2 weeks for the low-dose treatment and $38.8 \%$ for the high-dose treatment.

\section{Medication acceptance}

Four dimensions of medication acceptance by the patient were assessed with the NAMA questionnaire: attitude (patient has

Table 2 Disease severity (CGI-S) of patients treated with orally disintegrating olanzapine tablet or standard-coated olanzapine tablet at baseline and final visit (Day 5, LOCF)

\begin{tabular}{|c|c|c|c|c|c|c|c|c|}
\hline \multirow{3}{*}{$\begin{array}{l}\text { CGI-S } \\
\text { Physician assessment }\end{array}$} & \multicolumn{4}{|c|}{ SOT } & \multicolumn{4}{|c|}{ ODT } \\
\hline & \multicolumn{2}{|c|}{ Baseline } & \multicolumn{2}{|c|}{ Final visit } & \multicolumn{2}{|c|}{ Baseline } & \multicolumn{2}{|c|}{ Final visit } \\
\hline & $\mathbf{N}$ & $\%$ & $\mathbf{N}$ & $\%$ & $\mathbf{N}$ & $\%$ & $\mathbf{N}$ & $\%$ \\
\hline Borderline ill (2) & 0 & 0 & 9 & 4.3 & 0 & 0 & 7 & 2.8 \\
\hline Mildly ill (3) & 2 & 1.0 & 32 & 15.5 & 0 & 0 & 25 & 10.1 \\
\hline Moderately ill (4) & 13 & 6.3 & 92 & 44.4 & 10 & 4.1 & 97 & 39.3 \\
\hline Markedly ill (5) & 89 & 43.0 & 63 & 30.4 & 78 & 31.6 & 81 & 32.8 \\
\hline Severely ill (6) & 95 & 45.9 & 8 & 3.9 & 137 & 55.5 & 29 & 11.7 \\
\hline Extremely ill (7) & 8 & 3.9 & 3 & 1.5 & 22 & 8.9 & 8 & 3.2 \\
\hline
\end{tabular}

Abbreviations: CGI-S, Clinical Global Impression, Severity; ODT, orally disintegrating olanzapine tablet; SOT, standard-coated olanzapine tablet; LOCF, last observation carried forward. 
Table 3 Relative effects in Clinical Global Impression Improvement (CGI-I) and Clinical Global Impression Severity (CGI-S) improvement by baseline CGI-S for orally disintegrating olanzapine tablet compared to standard-coated olanzapine tablet

\begin{tabular}{|c|c|c|c|c|}
\hline & & \multicolumn{3}{|c|}{ Final visit (2 weeks) } \\
\hline & & Relative effect & \multicolumn{2}{|c|}{$\mathbf{9 5} \%$ confidence interval } \\
\hline & \multicolumn{2}{|l|}{ Baseline } & lower & upper \\
\hline$\overline{C G I-I}$ & $(n=454)$ & 0.5085 & 0.4587 & 0.5584 \\
\hline \multirow[t]{4}{*}{ CGI-S } & $\leq 4(n=25)$ & 0.6233 & 0.4110 & 0.8355 \\
\hline & $5(n=167)$ & 0.4784 & 0.3967 & 0.5601 \\
\hline & $6(n=232)$ & 0.4338 & 0.3626 & 0.5050 \\
\hline & $7(n=30)$ & 0.2159 & 0.0161 & 0.4158 \\
\hline
\end{tabular}

Abbreviations: CGI-I, Clinical Global Impression, Improvement, CGI-S, Clinical Global Impression, Severity; ODT, orally disintegrating olanzapine tablet; SOT, standardcoated olanzapine tablet.

a positive attitude towards medication), compliance: (patient complies with medication intake), ingestion (patient ingests medication), and nursing (no extensive nursing effort is needed to administer medication). Proportions of positive assessment (applies very much, applies) for medication acceptance questions are shown for baseline and final assessments after 2 weeks in Figure 3.
At baseline, medication acceptance by the patient was lower in patients treated with ODT, in particular regarding attitude (SOT 58.0\%, ODT 31.6\%), ingestion (SOT 75.4\%, ODT $48.9 \%$ ), and nursing (SOT $81.1 \%$, ODT 53.9\%). After 2 weeks, medication acceptance improved for both formulations, in particular for ODT (attitude SOT 78.3\%, ODT 68.4\%; ingestion SOT $89.4 \%$, ODT $83.4 \%$; nursing SOT $92.8 \%$, ODT $86.2 \%$ ).

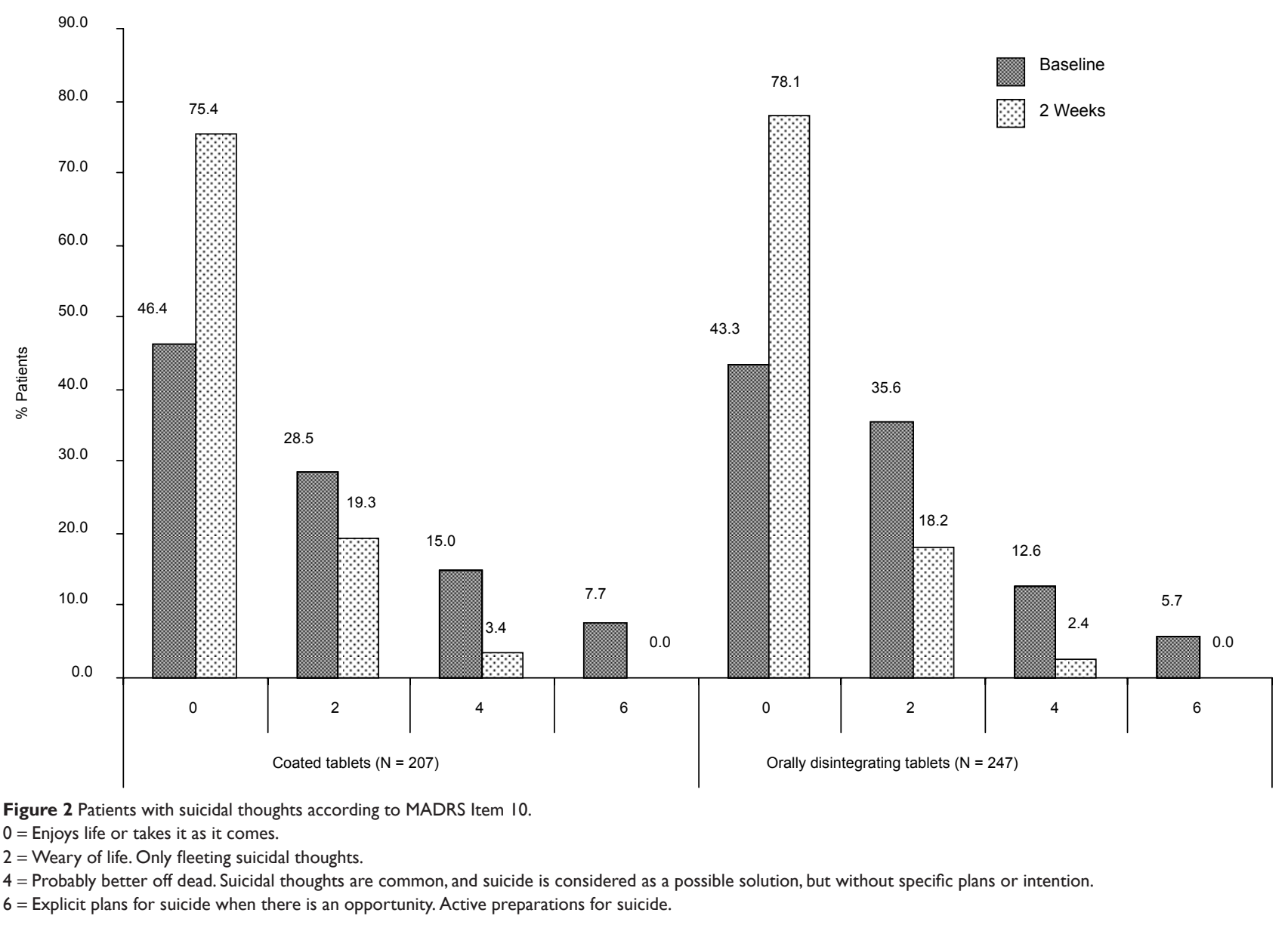




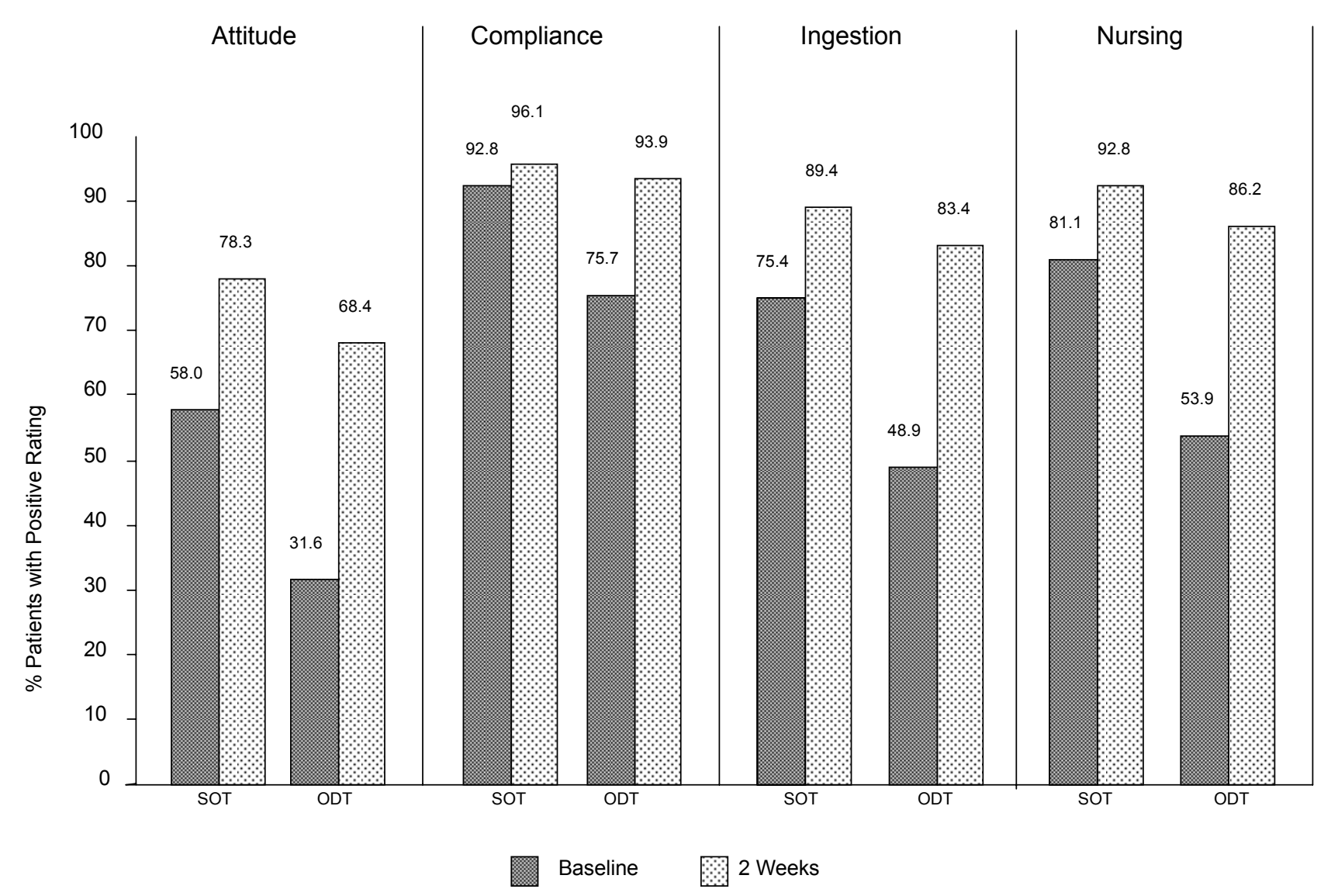

Figure 3 Patients with positive medication acceptance as rated by nursing personnel at baseline and final documentation (2 weeks). ODT, patients treated with orally disintegrating olanzapine tablets; SOT, patients treated with standard (coated) olanzapine tablets.

\section{Safety}

No deaths or serious adverse events were reported during this study. A total of $23(5.0 \%)$ patients experienced adverse events, and in 21 of these patients adverse events were considered as related to olanzapine treatment by the investigator (including not assessable and missing). The proportion of reported adverse events in patients receiving ODT was higher compared to patients receiving SOT $(6.5 \%$ vs $2.9 \%$, respectively).

The majority of the 28 adverse events documented in 23 patients were either of mild $(50.0 \%, 14 / 28)$ or moderate $(46.4 \%, 13 / 28)$ severity and only one adverse event $(3.6 \%)$ was considered as severe. In most cases patients recovered from their adverse events $(60.7 \%, 17 / 28)$, whereas $25.0 \%$ $(7 / 28)$ of events were not yet recovered by the end of the observation. The most frequently reported preferred term was weight gain $(2.0 \%, 9 / 456$ for all patients; $1.0 \%, 2 / 207$ for SOT; $2.8 \%, 7 / 247$ for ODT). Other adverse events reported in more than one (but in less than $1.0 \%$ ) patient were tiredness, extrapyramidal symptoms, vertigo, and nausea. Three patients $(0.7 \%, 3 / 456)$ discontinued prematurely because of an adverse event; they presented with an allergic reaction, an increase in creatinine kinase levels, and onset of restlessness (all of moderate intensity), respectively. All 3 received the ODT formulation.

\section{Discussion}

The objective of this study was to evaluate the use, effectiveness and tolerability of orally disintegrating olanzapine tablets (ODT) and standard (coated) olanzapine tablets (SOT) in patients admitted to psychiatric hospitals for emergency treatment. For the current study, effectiveness was defined as efficacy in the context of a natural setting.

Limitations of this prospective, open label study include the lack of randomization regarding the effectiveness comparisons, the frequent use of concomitant medications in a naturalistic setting, and a probable underreporting of adverse events in clinical practice compared to clinical trials. Besides, only patients who actually took oral medication could be assessed. Information about the rate of rejections or forced use of oral medication is not available. Finally, patients are described as using ODT or SOT based on the 
formulation they started with. No data are available if any patients switched to the other formulation during the 14-day observation period.

However, acknowledging these limitations, the naturalistic design provides a unique method of collecting information on use patterns and effectiveness in the clinical setting without the artefacts of an experimental intervention, in particular, as the patients observed receive acute treatment, for which only limited data from randomized clinical trials are obtainable.

Of particular interest in this study was the comparison of treatment patterns and medication acceptance with the two different oral olanzapine formulations (ie, either SOT or ODT). Major differences were found in patient characteristics, indicating that externally aggressive patients and those referred to inpatient treatment from 24-hour emergency services of psychiatric care hospitals (institutionalized patients), ie, those thought to refuse their antipsychotic medication, were preferably treated with ODT. Furthermore, patients treated with ODT received on average higher doses of olanzapine during the observation and were more severely ill at baseline. Therefore, in current clinical practice in Germany, acutely ill psychiatric patients receiving olanzapine are more commonly treated with ODT if they appear more severely ill and/or aggressive.

Although these differences in baseline characteristics are present, the results of the present study indicate that patients treated with SOT and ODT show similar shortterm (2-week) effectiveness in the treatment of psychiatric emergency patients with schizophrenia and related disorders in clinical practice.

The rating of medication acceptance (Figure 3) shows that ODT is preferably given to patients with a negative attitude towards medication and who are, therefore, at risk of being non-compliant, but still can be persuaded to accept oral medication. During the first 2 weeks of treatment, patients' attitude towards medication improved, particularly in the ODT cohort. Successful use of ODT in acutely noncompliant schizophrenic patients has already been reported for 85 patients in a controlled clinical trial (Kinon et al 2003). Prescription data gathered in the UK (Johnson et al 2002) suggest that olanzapine orally disintegrating tablets are frequently used as a treatment alternative to depot neuroleptics in patients with compliance problems.

Several studies suggested that clozapine and olanzapine improve suicidality in schizophrenic patients (Meltzer et al 1995; Beasley et al 1998; Meltzer et al 2003). Factors leading to a decrease in suicidality associated with clozapine or olanzapine may be improved cognitive function, reduced negative symptoms, and improved compliance. The results of the present study show that in patients treated with both olanzapine formulations suicidality (suicidal thoughts/ideation) decreased to a similar extent, indicating that the orally disintegrating tablet may also be considered in the treatment of acutely ill schizophrenic patients with either self-directed (suicidal) or externally-directed aggression. Compared with conventional antipsychotics (eg, haloperidol), preliminary evidence suggests that atypical antipsychotics effectively reduce agitation, are better tolerated, and are associated with fewer adverse effects (Wright et al 2001; Breier et al 2002; Krakowski et al 2006; Marder 2006). In our study, the rate of adverse events reported in clinical practice was low and both formulations were well tolerated. However, the rate of adverse events was higher in patients receiving ODT compared to those receiving SOT. This may reflect baseline differences in patient characteristics: ODT was preferably used in mostly aggressive patients with more severe clinical symptomatology at baseline. This may have contributed to the observed difference in reported adverse events.

A considerable proportion $(>50 \%)$ of the acutely ill psychiatric patients in this naturalistic study were treated with high doses (ie, $\geq 20 \mathrm{mg}$ /day) of olanzapine. However, no major differences in treatment outcome or tolerability between these dose groups defined post-hoc were seen. Further, no differences in the rate or type of adverse events were reported for patients who received low-dose vs high-dose olanzapine treatment. This is in line with several previous randomized controlled studies which have provided evidence for a safe and effective use of high-dose olanzapine in patients with severe acute and/or treatment resistant schizophrenia (Tollefson et al 2001; Volavka et al 2002; Baker et al 2003; Krakowski et al 2006). One recent randomized, double-blind controlled study has directly compared standard and higher doses of olanzapine (10 mg, $20 \mathrm{mg}, 40 \mathrm{mg}$ ) in patients with acute schizophrenia and schizoaffective disorders (Kinon et al 2006). The results suggested that most individuals in the general population of patients with schizophrenia or schizoaffective disorders will respond to the standard olanzapine dose range, but the use of higher doses may be warranted for a more severely ill patient population. A recent naturalistic study in 80 patients with severe agitation conducted in Spain also found significant improvements on the PANSS-EC and Agitation-Calmness Evaluation scale (ACES) following a $20 \mathrm{mg}$ dose of olanzapine ODT. Treatment was well-tolerated and no serious treatment-emergent adverse events were observed (Pascual et al 2007). Our results nevertheless 
demonstrate that in current clinical practice, high-dose treatment in patients with acute schizophrenia is common.

\section{Conclusions}

In conclusion, this non-randomized, observational study shows comparable outcomes and good tolerability in patients treated with both oral formulations of olanzapine. In an acute emergency setting, orally disintegrating tablets were preferably used for more severely ill and aggressive patients with low medication acceptance and were used in slightly higher doses compared to coated tablets.

\section{Acknowledgments}

This study was supported by Lilly Deutschland $\mathrm{GmbH}$, Bad Homburg, Germany. We thank Frank Kessler, Lilly Deutschland $\mathrm{GmbH}$, for conducting statistical analyses, and Karin Helsberg, Lilly Deutschland $\mathrm{GmbH}$, for editing and proofreading of the manuscript.

This paper was partially presented at the Eleventh Biennial Winter Workshop on Schizophrenia, February 7-14, 2004, Davos, Switzerland.

\section{List of abbreviations}

CGI-I, Clinical Global Impression - Improvement; CGI-S, Clinical Global Impression - Severity; NGI-I, Nurse Global Impression - Improvement; NGI-S, Nurse Global Impression - Severity; MADRS, Montgomery Åsberg Depression Rating Scale; NAMA, Nursing assessment of medication acceptance scale; ODT, Orally disintegrating olanzapine tablets; SOT, standard-coated tablets.

\section{References}

Alvarez E, Bobes J, Gomez J-C, et al. 2003. Safety of olanzapine versus conventional antipsychotics in the treatment of patients with acute schizophrenia. A naturalistic study. Eur Neuropsychopharmacol, 13:39-48.

Baker RW, Kinon BJ, Maguire GA, et al. 2003. Effectiveness of rapid initial dose escalation of up to forty milligrams per day of oral olanzapine in acute agitation. J Clin Psychopharmacol, 23:342-8.

Beasley CM, Sayler ME, Kiesler GM. 1998. The influence of pharmacotherapy on self-directed and externally-directed aggression in schizophrenia. Schizophr Res, 29:28.

Behnke, K, Sogaard J, Martin S, et al. 2003. Mirtazapine orally disintegrating tablet versus sertaline: a prospective onset of action study. $J$ Clin Psychopharmacol, 23:358-64.

Bergstrom RF, Mitchell M, Witcher J, et al. 2004. Rapid onset of absorption with olanzapine orally disintegrating tablets. Schizophr Res 67:160.

Bobes J, Gibert J, Ciudad A, et al. 2003. Safety and effectiveness of olanzapine versus conventional antipsychotics in the acute treatment of first-episode schizophrenic inpatients. Prog Neuropsychopharmacol Biol Psychiatry 27:473-81.

Breier A, Meehan K, Birkett M, et al. 2002. A double-blind, placebocontrolled dose-response comparison of intramuscular olanzapine and haloperidol in the treatment of acute agitation in schizophrenia. Arch Gen Psychiatry 59:441-8.
Brunner E, Domhof S, Langer F. 2002. Nonparametric analysis of longitudinal data in factorial experiments. Wiley Series in Probability and Statistics. New York: Wiley.

Brunner E, Munzel U. 2002. Nichtparametrische Datenanalyse. Berlin: Springer.

Cain ED. 2006. Clinical perspectives on atypical antipsychotics for treatment of agitation. J Clin Psychiatry 67 (Suppl 10):22-31.

Chue P, Jones B, Taylor CC, Dickson R. 2002. Dissolution profile, tolerability, and acceptability of the orally disintegrating olanzapine tablet in patients with schizophrenia. Can J Psychiatry, 47:771-4.

Cooper D, Moisan J, Grégoire JP. 2007. Adherence to atypical antipsychotic treatment among newly treated patients: a population-based study in schizophrenia. J Clin Psychiatry 68:818-25.

Dowson AJ, MacGregor EA, Purdy RA, et al. 2002. Zolmitriptan orally disintegrating tablet is effective in the acute treatment of migraine. Cephalalgia 22:101-6.

Glazer WM. 1998. Formulary decision and health economics. J Clin Psychiatry 59(Suppl):23-9.

Guy W. 1976. ECDEU Assessment Manual for Psychopharmacology (revised). Bethesda, DM: US Department of Health, Education and Welfare.

Johnson D, Rai S, Milne S. 2002. Olanzapine oro-dispersible (Velotab ${ }^{\mathrm{TM}}$ ) - an alternative to depot? Int J Psychiatry Clin Pract 6:163-5.

Kinon BJ, Hill AL, Liu H, Kollack-Walker S. 2003. Olanzapine orally disintegrating tablets in the treatment of acutely ill non-compliant patients with schizophrenia. Int J Neuropsychopharmacol 6:97-102.

Kinon BJ, Volavka J, Stauffer V, et al. 2006. Standard and higher doses of olanzapine in acutely ill patients with schizophrenia or schizoaffective disorder with suboptimal prior response: a randomized double-blind fixed dose study. [Abstract]. Int J Neuropsychopharmacol 9 (Suppl 1):S281.

Krakowski MI, Czobor P, Citrome L, et al. 2006. Atypical antipsychotic agents in the treatment of violent patients with schizophrenia and schizoaffective disorder. Arch Gen Psychiatry, 63:622-9.

Marder SR. 2006. A review of agitation in mental illness: treatment guidelines and current therapies. J Clin Psychiatry, 67 (Suppl 10):13-21

Meltzer HY, Alphs L, Green AI, et al. 2003. Clozapine treatment for suicidality in schizophrenia: International Suicide Prevention Trial (InterSePT). Arch Gen Psychiatry, 60:82-91.

Meltzer HY, Okayli G. 1995. Reduction of suicidality during clozapine treatment of neuroleptic-resistant schizophrenia: impact on risk-benefit assessment. Am J Psychiatry, 152:183-90.

Montgomery SA, Asberg M. 1979. A new depression scale designed to be sensitive to change. Br J Psychiatry, 134:382-9.

Pascual JC, Pérez V, Martín JL, Safont G, Puigdemont D, Alvarez E. 2007. Olanzapine orally-disintegrating tablet in severe psychotic agitation: a naturalistic study. Actas Esp Psiquiatr, 35:47-51.

Soskis DA. 1978. Schizophrenic and medical inpatients as informed drug consumers. Arch Gen Psychiatry, 35:645-7.

Tollefson GD, Birkett MA, Kiesler GM, Wood AJ. Lilly Resistant Schizophrenia Study Group. 2001. Double-blind comparison of olanzapine versus clozapine in schizophrenic patients clinically eligible for treatment with clozapine. Biol Psychiatry, 49:52-63.

Torrey EF. 1995. Surviving schizophrenia: A manual for families, consumers, and providers. New York: Harper Collins. p 409.

Volavka J, Czobor P, Sheitman B, et al. 2002. Clozapine, olanzapine, risperidone, and haloperidol in the treatment of patients with chronic schizophrenia and schizoaffective disorder. Am J Psychiatry, 159:255-62.

Wright P, Birkett M, David SR, et al. 2001. Double-blind, placebo-controlled comparison of intramuscular olanzapine and intramuscular haloperidol in the treatment of acute agitation in schizophrenia. Am J Psychiatry 158:1149-51. 
